\title{
CLINICAL SECTION RUPTURE OF A SCROTAL HERNIA
}

By R. Marcus, M.D., Ch.M., F.R.C.S.

Rupture of the small intestine in a hernial sac is not common. It may result from reduction by taxis of a strangulated hernia or nipping of the bowel between the pubis and a badly fitting truss (Reid, 1948).

Case Report. T.J.J., aet. 74, a retired farmer, had a left scrotal hernia for 10 years. On $8 / 6 / 51$ it became painful and he was not able to reduce it. Two hours later the patient's practitioner reduced the hernia without difficulty but immediately following this procedure the patient collapsed with severe central abdominal pain. Two hours later he was admitted to hospital, and one hour later operated upon.

On Examination. The patient was in obvious pain. His extremities were cold and cyanosed. Temperature, $97^{\circ}$ F. Pulse, r20 per minute. Respirations, 22 per minute; systolic blood pressure, $50 \mathrm{~mm}$. 'Hg. The hernia had been reduced completely. The abdomen did not move with respiration and was scaphoid. There was generalized board-like rigidity with marked rebound tenderness in the left side of the hypogastrium. Rectal examination revealed no abnormality.

Operation. Under intravenous pethidine and adrenalin drip $(1 / 250,000)$ anaesthesia a left paramedian incision was made. Intestinal contents welled out of the wound. On opening the peritoneal cavity several matted coils of distended and thickened small intestine were delivered into the wound. On the antimesenteric border of one of the coils there was a laceration about an inch long. The laceration was closed with interrupted mucosa to mucosa sutures and then oversown with a continuous catgut suture. No attempt was made to separate the loops, which were firmly bound together by old adhesions. The bowel was then replaced in the peritoneal cavity, which was drained by a split tube and wick placed over the suture line.

Post-Operative Progress. The gastric contents were aspirated hourly for 48 hours. Intravenous glucose saline, 4 litres a day, was given for 3 days: Penicillin, 250,000 units 6-hourly, given intravenously for 7 days and soluble sulphathiazole, I gram stat and $\frac{1}{2}$ gram 6-hourly, intravenously for 3 days and then orally for 2 days. Apart from retention of urine, which was relieved by catheterization, the patient had an uneventful convalescence. A straight $\mathrm{X}$-ray of the abdomen on $2 / 7 / 5$ I showed no fluid levels in the small bowel.

Remarks, The patient had an idiosyncrasy to pethidine which was given as premedication. This rendered him sufficiently analgesic for the operation to be carried out without other anaesthetics. Owing to the poor general condition, no attempt was made to explore the abdomen or relieve the chronic distension in the loops of bowel reduced from the hernial sac. There is no direct evidence that the bowel was ruptured by the manipulations before admission to hospital. The fact that it teduced easily is against this possibility. It may be that the distended bowel ruptured before this procedure and when it was reduced the intestinal contents poured freely into the general peritoneal cavity. Another possibility was perforation by a foreign body which was missed because no attempt was made to locate it.

Summary. A case of rupture of a loop of chronically distended small intestine in a hernial sac is reported. The patient recovered after suture of the lacerated bowel.

REID, D. R. K. (1948) Brit. Med. Ұnl. 2, 824 